\title{
Function Theories in Cayley-Dickson Algebras and Number Theory
}

\author{
Rolf Sören Kraußhar
}

\begin{abstract}
In the recent years a lot of effort has been made to extend the theory of hyperholomorphic functions from the setting of associative Clifford algebras to non-associative Cayley-Dickson algebras, starting with the octonions.

An important question is whether there appear really essentially different features in the treatment with Cayley-Dickson algebras that cannot be handled in the Clifford analysis setting. Here we give one concrete example: Cayley-Dickson algebras admit the construction of direct analogues of so-called CM-lattices, in particular, lattices that are closed under multiplication.

Canonical examples are lattices with components from the algebraic number fields $\mathbb{Q}\left[\sqrt{m_{1}}, \ldots \sqrt{m_{k}}\right]$. Note that the multiplication of two non-integer lattice paravectors does not give anymore a lattice paravector in the Clifford algebra. In this paper we exploit the tools of octonionic function theory to set up an algebraic relation between different octonionic generalized elliptic functions which give rise to octonionic elliptic curves. We present explicit formulas for the trace of the octonionic CM-division values.
\end{abstract}

Mathematics Subject Classification (2010). Primary 30G35; Secondary 11G15.

Keywords. Function theory in Cayley-Dickson algebras, generalized elliptic functions, generalized CM lattices, algebraic number fields, octonions.

\section{Introduction}

There are a number of different possibilities to generalize complex function theory to higher dimensions.

One classical and well-established option is to consider functions in several complex variables in $\mathbb{C}^{n}$ where the classical holomorphicity concept is applied separately to each complex variable, see, for example, $[8,15]$. From the viewpoint of algebraic geometry, the theory of several complex variables provides the natural setting to study Abelian varieties and curves.

Another possibility is offered by Clifford analysis which considers null-solutions to a generalized Cauchy-Riemann operator that are defined in a subset of vectors or 
paravectors and that take values in an associative Clifford algebra, see, for instance, $[3,5,12,21]$.

In the recent years one also observes a lot of progress in extending the constructions from the setting of associative Clifford algebras to non-associative CayleyDickson algebras, in particular, to the framework of octonions, see, for example, $[7,9,11,17,18,19,25]$.

Although one has no associativity anymore, at least in the octonionic case it was possible to also generalize a number of classical theorems, such as the Cauchy integral formula or the formulas for the Taylor and Laurent series representations by following more or less the same line of argumentation as performed in Clifford analysis. See $[16,26,30,31,32,33]$. Due to the non-associativity one has to bracket terms in a particular way together. However, apart from this, some of the results still look very similar to the formulas derived for the Clifford algebra case, at least at a first glance.

Therefore, an important question is whether there appear really essentially different features in the treatment with the more complicated non-associative CayleyDickson algebras. In [18] and [19] the authors present some important structural differences. In contrast to the Clifford analysis setting, octonionic regular functions in the sense of the Riemann approach, called $\mathbb{O}$-regular for short, do not form a left or right $\mathbb{O}$-module anymore. If $f$ is a left $\mathbb{O}$-regular function, then it is not guaranteed that $f \lambda$ is also left $\mathbb{O}$-regular. So, there is no one-to-one correspondence between the set of monogenic functions in $\mathbb{R}^{8}$ embedded in an associative Clifford algebra $C l_{07}$ and $\mathbb{O}$-regular functions.

On the one hand, the lack of this property represents an obstacle in the development of generalizations of many other theorems to the non-associative setting. On the other hand, there is the challenge to figure out problems that really require a treatment with non-associative Cayley-Dickson algebras that cannot be treated in the Clifford analysis setting.

One aim of this paper to describe one very concrete example.

Cayley-Dickson algebras $\mathcal{C}_{k}$ offer the possibility to consider lattices $\Omega \subset \mathcal{C}_{k}$ that admit non-trivial left and right ideals $\mathcal{L}$ and $\mathcal{R}$ such that $\mathcal{L} \Omega \subseteq \Omega$, resp. $\Omega \mathcal{R} \subseteq$ $\Omega$. These are natural generalizations of the so-called classical CM-lattices (lattices that remain invariant under multiplication by some non-trivial non-integer complex numbers). These lattices play an important role in the treatment of algebraic points of elliptic curves, cf. [22]. In particular, in Cayley-Dickson algebras one can consider lattices which, apart from their algebraic structure of a module, are additionally closed under multiplication. See also [1] in which some basic properties of generalized CM-lattices in some graded $\mathbb{R}_{F} \mathbb{Z}^{n}$-algebras with deformations have been discussed, in particular, for $\mathbb{R}_{F} \mathbb{Z}^{3}$-algebras and Clifford algebras.

In fact, one can consider CM-lattices in Clifford algebras. However, Clifford analysis is restricted to considering functions that are only defined in the subset 
of paravectors and not for variables from the full Clifford algebra (apart from the particular quaternionic case). Note, however, that the multiplication of two noninteger lattice paravectors from $\mathbb{R}^{n+1}$ does not give anymore a lattice paravector from $\mathbb{R}^{n+1}$. It gives an element from the full Clifford algebra that involves bivector parts. Therefore, a monogenic function evaluating CM-values of the form $f(\mu \omega)$ with non-real multiplicators $\mu \in \mathcal{L}$ and non-real lattice paravectors $\omega \in \Omega$ cannot be defined. In the framework of Clifford analysis, the argument in the function must again be a paravector. In [20] we introduced a two-sided kind of CM-multiplication, considering a simultaneous multiplication by the same multiplicator from the left and from the right to the lattice of the form $f(\eta \omega \eta)$. In fact, whenever $\omega$ and $\eta$ are paravectors, then the special product of the form $\eta \omega \eta$ actually again gives a paravector. But this is not the case if one applies the multiplicator $\eta$ only from one side or if one considers on both sides of $\omega$ two different multiplicators $\eta$ and $\mu$. In these cases one has no paravector anymore, in general.

This obstacle can successfully be overcome using Cayley-Dickson algebras instead. In contrast to the Clifford algebra setting, in the octonions every non-zero element is invertible.

More generally, in the context of Cayley-Dickson algebras, one can meaningfully define functions where the arguments may stem from subsets of the full CayleyDickson algebra and not only from the subset of paravectors.

Now, the function theory in Cayley-Dickson algebras also admits the construction of generalized Weierstraß elliptic functions which satisfy the regularity criterion in the sense of the Riemann approach. Taking special care of the non-associativity, the construction of the regular analogue of the Weierstraß $\wp$-function can be performed in exactly the same way as in Clifford analysis (cf. [21], [27]), namely by periodizing the partial derivatives of the associated $\mathbb{O}$-regular Cauchy kernel and adding some convergence preserving terms. This possibility was already roughly outlined in [20, 21]. Recently, a 7-fold periodic generalization of the cotangent series was explicitly written out particularly for the octonionic case in [25]. This also underlines the current interest in this topic.

However, the treatment with Cayley-Dickson algebras provides us with a new feature, if we consider these functions in association with a period lattice that has a non-trivial one-sided Cayley-Dickson multiplication, since we do not have such an algebraic structure in the Clifford analysis setting where we are restricted to define the functions on the space of paravectors.

At least in the octonionic cases we are able to deduce explicit algebraic relations between the different CM-division values of some prototypes of generalized elliptic functions. The canonical examples of CM-lattices in Cayley-Dickson algebras are lattices whose components stem from multi-quadratic number fields $\mathbb{Q}\left[\sqrt{m_{1}}, \ldots \sqrt{m_{k}}\right]$. In the octonionic case the canonical examples are tri-quadratic number fields. This is also in line with the classical complex case in which we deal with imaginary quadratic number fields of the form $\mathbb{Q}[\sqrt{-m}]$. 
The paper is structured as follows.

In Section 2, we summarize the most important facts and notions about hypercomplex numbers in Cayley-Dickson algebras and recall their basic properties which are used in the sequel of this paper.

In Section 3, we look at integral domains and introduce lattices with CayleyDickson multiplications. We describe them in terms of generalized integrality conditions involving norms and trace expressions. We show that lattices of the form $\mathbb{Z}+\sum_{i=1}^{2^{k}-1} \mathbb{Z}_{i} \omega_{i}$, where the real components of the primitive periods stem from an algebraic field of the form $\mathbb{Q}\left[\sqrt{m_{1}}, \ldots \sqrt{m_{k}}\right]$ and where the elements $m_{1}, \ldots, m_{k}$ are all mutually distinct positive square-free integers, serve as important non-trivial examples of lattices with Cayley-Dickson multiplication.

In Section 4, we give a short overview on which basic tools can be carried over from Clifford analysis to the non-associative setting and explain where we meet structural obstacles. While generalizations of the Weierstraß $\zeta$-function and $\wp$-function can even be introduced in general Cayley-Dickson algebras, a number of structural and technical features require at least an alternative or a composition algebra. So we turn to focus on octonions in the sequel.

The core piece of the paper is Section 4.3, where we establish algebraic relations between the values of the octonionic generalized Weierstraß $\zeta$-function at a point and their octonionic CM-division values. In particular, we present an explicit algebraic formula to calculate the trace of the octonionic division values of the generalized octonionic regular Weierstraß $\wp$-function. They turn out to be elements of the number field generated by the algebraic elements of the lattice components and the components of the Legendre-constants which still require an algebraic investigation in the future.

An exciting topic of future research in this direction would be to ask whether the octonionic functions can be used to do some class field theory. Do these division values play some role in the construction of Galois field extensions of the abovementioned number fields?

The Jugendtraum of Kronecker addressing the classification of all abelian field extensions of imaginary quadratic number fields $\mathbb{Q}[\sqrt{-m}]$ is an extension of the famous Kronecker-Weber theorem stating that all abelian extensions of $\mathbb{Q}$ are contained in cyclotomic extensions.

The solution of Kronecker's Jugendtraum was the motivation for the development of class field theory which took more than 70 years (starting from D. Hilbert's famous Zahlbericht [14] and continuing with P. Furtwängler, H. Weber, T. Tagaki, E. Artin, K. Hey, M. Zorn, M. Matchett, and many others, among them also R. Fueter, who was a student of D. Hilbert).

It turned out that the complex division values of the associated meromorphic doubly periodic function $\frac{g_{2} g_{3}}{g_{2}^{3}-27 g_{3}^{2}} \wp(z)$ lie in abelian Galois field extensions of $\mathbb{Q}[\sqrt{-m}]$, cf. [28], similarly as the division values of the exponential functions lie in abelian extensions of $\mathbb{Q}$. 
The construction of algebraic field extensions of bi-quadratic number fields was a crucial motivation for R. Fueter to develop hypercomplex function theories. He developed his four-fold periodic monogenic quaternionic elliptic functions exactly for this purpose, cf. [10, 23]. The framework of Cayley-Dickson algebras now even addresses more generally than quaternions multi-quadratic number fields containing imaginary quadratic number fields and also biquadratic number fields considered by R. Fueter as special subcases, among many others. However, to address multiquadratic extensions of $\mathbb{Q}$ one first needs to develop a Kummer theory. Even the case of biquadratic extensions involves additional difficulties arising from the splitting of primes. The question whether one can do some class field theory with octonions hence definitely represents an exciting open research field.

Explicit formulas for the division values of generalized multiperiodic functions that we developed in this paper might have some interest in this sense, but there are also many fundamental open problems that need to be solved in order to be able to give some satisfactory answers to this question.

\section{Some basic properties of Cayley-Dickson algebras}

We start by introducing the construction principle of Cayley-Dickson algebras. For details, we refer the reader to, for instance, [1, 2, 16, 29] and elsewhere. They contain all normed real division algebras, i.e., the fields of real and complex numbers $\mathbb{R}$ and $\mathbb{C}$, the skew field of Hamiltonian quaternions $\mathbb{H}$, and the non-associative alternative octonions $\mathbb{O}$ as special cases.

Following [29], one may start with a ring $R$ that has a two-sided multiplicative neutral element 1 and a non-necessarily commutative and non-necessarily associative multiplication. Furthermore, we impose that it has a "conjugation" antiautomorphism $a \mapsto \bar{a}$ with the properties that $\overline{a+b}=\bar{a}+\bar{b}, \overline{a b}=\bar{b} \bar{a}$, and $\overline{\bar{a}}=a$ for all $a, b \in R$. Then one forms pairs of numbers of the form $(a, b)$ and $(c, d)$ and defines an addition and multiplication operation by

$$
(a, b)+(c, d):=(a+c, b+d), \quad(a, b) \cdot(c, d):=(a c-d \bar{b}, \bar{a} d+c b) .
$$

The conjugation is extended by $\overline{(a, b)}:=(\bar{a},-b)$.

The simplest choice is to take for the ring $R$ the real numbers $\mathbb{R}$ in which we have $\bar{a}=a$. The above-indicated doubling process, called Cayley-Dickson doubling, then generates in the first step the complex number field $\mathbb{C}$. It is the first Cayley-Dickson algebra generated by the doubling process starting with $\mathbb{R}$. If we continue performing this doubling procedure, then we obtain a chain of non-necessarily commutative nor associative algebras which are the (classical) Cayley-Dickson algebras $\mathcal{C}_{k}$ where $k$ denotes the step of the doubling procedure. The next Cayley-Dickson algebra $\mathcal{C}_{2}$ is the skew field of Hamiltonian quaternions, where each element can be written in the form $z=x_{0}+x_{1} e_{1}+x_{2} e_{2}+x_{3} e_{3}$. Here, $e_{i}^{2}=-1$ for $i=1,2,3, e_{1} e_{2}=e_{3}$, $e_{2} e_{3}=e_{1}, e_{3} e_{1}=e_{2}$, and $e_{i} e_{j}=-e_{j} e_{i}$ for all distinct $i, j$ from $\{1,2,3\}$. This algebra is not commutative anymore, but it is still associative. It is still an example of an associative Clifford algebra. This is not anymore the case after having performed 
the following doubling step where we arrive at the octonions $\mathbb{O}$. Octonions have the form

$$
z=x_{0}+x_{1} e_{1}+x_{2} e_{2}+x_{3} e_{3}+x_{4} e_{4}+x_{5} e_{5}+x_{6} e_{6}+x_{7} e_{7}
$$

where $e_{4}=e_{1} e_{2}, e_{5}=e_{1} e_{3}, e_{6}=e_{2} e_{3}$, and $e_{7}=e_{4} e_{3}=\left(e_{1} e_{2}\right) e_{3}$. Like in the quaternionic case, we have $e_{i}^{2}=-1$ for all $i=1, \ldots, 7$ and $e_{i} e_{j}=-e_{j} e_{i}$ for all mutually distinct $i, j \in\{1, \ldots, 7\}$. The multiplication is visualized in the following multiplication table:

\begin{tabular}{|l|rrrrrrr|}
$\cdot$ & $e_{1}$ & $e_{2}$ & $e_{3}$ & $e_{4}$ & $e_{5}$ & $e_{6}$ & $e_{7}$ \\
\hline$e_{1}$ & -1 & $e_{4}$ & $e_{5}$ & $-e_{2}$ & $-e_{3}$ & $-e_{7}$ & $e_{6}$ \\
$e_{2}$ & $-e_{4}$ & -1 & $e_{6}$ & $e_{1}$ & $e_{7}$ & $-e_{3}$ & $-e_{5}$ \\
$e_{3}$ & $-e_{5}$ & $-e_{6}$ & -1 & $-e_{7}$ & $e_{1}$ & $e_{2}$ & $e_{4}$ \\
$e_{4}$ & $e_{2}$ & $-e_{1}$ & $e_{7}$ & -1 & $-e_{6}$ & $e_{5}$ & $-e_{3}$ \\
$e_{5}$ & $e_{3}$ & $-e_{7}$ & $-e_{1}$ & $e_{6}$ & -1 & $-e_{4}$ & $e_{2}$ \\
$e_{6}$ & $e_{7}$ & $e_{3}$ & $-e_{2}$ & $-e_{5}$ & $e_{4}$ & -1 & $-e_{1}$ \\
$e_{7}$ & $-e_{6}$ & $e_{5}$ & $-e_{4}$ & $e_{3}$ & $-e_{2}$ & $e_{1}$ & -1 \\
\hline
\end{tabular}

As one can easily deduce with the help of this table, the octonions are not associative anymore. Therefore, they are no Clifford algebras anymore. However, one still has a number of nice properties, stemming from the fact that the octonions still form an alternative composition algebra.

In particular, one has the Moufang relations, guaranteeing that

$$
(a b)(c a)=a((b c) a)
$$

for all $a, b, c \in \mathbb{O}$, which, in particular, for $c=1$ gives the flexibility condition $(a b) a=a(b a)$. Moreover, one has the important rule

$$
(a \bar{b}) b=\bar{b}(b a)=a(\bar{b} b)=a(b \bar{b})
$$

for all $a, b \in \mathbb{O}$.

All the first Cayley-Dickson algebras $\mathcal{C}_{k}$ with $k \leq 3$ are division algebras.

In the next step of the Cayley-Dickson doubling we then obtain the 16-dimensional sedenions. As mentioned in [16], one has $e_{j} e_{k}=-\delta_{j k}+\varepsilon_{j k m} e_{m}$, where $\delta_{i j}$ is the usual Kronecker symbol and $\varepsilon_{j k m}$ is the usual epsilon tensor, which is totally antisymmetric in its indices given by the usual permutation rule for a 3 -indexed antisymmetric tensor with values from $\{0,1,-1\}$.

Up from here we have to deal with zero divisors (which in the case of sedenions is a measure zero subset) and one even loses the alternative multiplication structure. Artin's theorem only guarantees the power associativity for $\mathcal{C}_{k}$ with $k \geq 4$. Therefore, we also lose the general Moufang identities at this level.

But an important property remains: that each element $z=x_{0}+\sum_{j=1}^{2^{k}-1} x_{j} e_{j}$ of a Cayley-Dickson algebra $\mathcal{C}_{k}$ satisfies a quadratic equation of the form

$$
z^{2}-\mathcal{S}(z) z+\mathcal{N}(z)=0
$$


where $\mathcal{S}(z)=z+\bar{z}=2 x_{0}$ is the trace and where

$$
\mathcal{N}(z)=z \bar{z}=|z|^{2}=\sum_{i=0}^{2^{k}-1} x_{i}^{2}
$$

is the norm of $z$, cf. [29]. Note that, in general, $\operatorname{dim}_{\mathbb{R}} \mathcal{C}_{k}=2^{k}$.

The only real normed division algebras, where one has the multiplicative property $\mathcal{N}(z w)=\mathcal{N}(z) \mathcal{N}(w)$, are $\mathbb{R}, \mathbb{C}, \mathbb{H}$, and $\mathbb{O}$. Up from the sedenions it can happen that $\mathcal{N}(z w)-\mathcal{N}(z) \cdot \mathcal{N}(w) \neq 0$, see $[16]$.

To conclude this section, we want to mention that we can get a different chain of algebras if we construct the doubling differently, as proposed, for instance, in [29]. In the framework of a different doubling it is possible to maintain some of the nicer properties, such as the multiplicativity of the norm.

\section{Integrality conditions and lattices with Cayley-Dickson multiplication}

In this section we introduce some number-theoretical concepts. We start with

Definition 3.1. An element $z$ from a Cayley-Dickson algebra $\mathcal{C}_{k}$ is called rational (resp. integral) if its trace $\mathcal{S}(z)$ and its norm $\mathcal{N}(z)$ are a rational number from $\mathbb{Q}$ (or an integer from $\mathbb{Z}$, respectively).

Now suppose that we deal with a set of rational or integral Cayley-Dickson numbers, respectively. If these numbers satisfy additionally

$$
\mathcal{N}(a+b), \mathcal{N}(a \cdot b), \mathcal{S}(a+b), \mathcal{S}(a \cdot b) \in \mathbb{Q}(\text { resp. } \in \mathbb{Z})
$$

for all $a, b$, then this set of Cayley-Dickson numbers forms a ring and even an algebra which, however, is not necessarily commutative nor associative. In the quaternionic setting, an algebra of rational (integral) quaternions that satisfy the abovementioned conditions is sometimes called a quaternionic rational (integral) Brandt algebra, cf. $[1,10]$. In exactly the same way one can define octonionic rational and integral Brandt algebras.

As in the quaternionic setting, also in the octonionic setting one can easily characterize multiplicatively invariant rational and integral Brandt algebras in the following way:

Proposition 3.2. Two rational (resp. integral) octonions $a, b \in \mathbb{O}$ belong to a rational (integral) non-associative Brandt algebra if and only if $2\langle a, b\rangle$ and $2\langle a, \bar{b}\rangle$ are elements from $\mathbb{Q}$ (resp. from $\mathbb{Z})$, where $\langle\cdot, \cdot\rangle$ is the usual Euclidean scalar product in $\mathbb{R}^{8}$.

Proof. In the octonions one still has

$$
\mathcal{N}(a b)=(a b) \cdot \overline{(a b)}=a b(\bar{b} \bar{a})=a(b \bar{b}) \bar{a}=\mathcal{N}(a) \mathcal{N}(b) .
$$


Furthermore,

$$
\mathcal{N}(a+b)=(a+b) \overline{(a+b)}=a \bar{a}+b \bar{b}+a \bar{b}+b \bar{a}=\mathcal{N}(a)+\mathcal{N}(b)+2\langle a, b\rangle .
$$

Next, we have

$$
\mathcal{S}(a b)=a b+\overline{a b}=2\langle a, \bar{b}\rangle,
$$

and trivially $\mathcal{S}(a+b)=\mathcal{S}(a)+\mathcal{S}(b)$.

Remark 3.3. If the ring is additionally stable under conjugation, then the second condition $2\langle a, \bar{b}\rangle \in \mathbb{Q}($ resp. $\in \mathbb{Z})$ can be dropped.

Next we introduce the concept of generalized complex multiplication of lattices in Cayley-Dickson algebras.

\section{Definition 3.4 (Lattices with Cayley-Dickson multiplication).}

Let $\Omega_{2^{k}}=\mathbb{Z} \omega_{0}+\mathbb{Z} \omega_{1}+\cdots+\mathbb{Z} \omega_{2^{k}-1}$ be a $2^{k}$-dimensional lattice, where all elements $\omega_{h}\left(h=0, \ldots 2^{k}-1\right)$ are $\mathbb{R}$-linearly independent elements from $\mathcal{C}_{k}$. Then we say that $\Omega_{2^{k}}$ has a left (right) $\mathcal{C}_{k^{-}}$-multiplication if there exists an $\eta \in \mathcal{C}_{k} \backslash \mathbb{Z}$ such that

$$
\eta \cdot \Omega_{2^{k}} \subseteq \Omega_{2^{k}}, \quad \text { resp. } \quad \Omega_{2^{k}} \cdot \eta \subseteq \Omega_{2^{k}} .
$$

In the case where we have $\eta \omega \in \Omega_{2^{k}}$ for all $\eta, \omega \in \Omega_{2^{k}}$ we say that the lattice is closed under multiplication. In this case, the lattice forms a non-associative ring.

In the more general case, the (non-associative) ring of left multiplicators form a left (resp. right) ideal. Lattices with Cayley-Dickson multiplication can be constructed by choosing the primitive generators from a rational or integral Brandt algebra. The most important examples are lattices whose components stem from multiquadratic number fields. The canonical examples can be constructed as follows.

Take $k$ mutually distinct square-free positive integers $m_{1}, \ldots, m_{k}$.

Then take a lattice of the form

$$
\mathbb{Z}+\mathbb{Z} \omega_{1}+\cdots+\mathbb{Z} \omega_{k}+\mathbb{Z} \omega_{1} \omega_{2}+\cdots+\mathbb{Z} \omega_{k-1} \omega_{k}+\cdots+\mathbb{Z}\left(\left(\omega_{1} \cdots \omega_{k-2}\right) \omega_{k-1}\right) \cdot \omega_{k},
$$

where

$$
\begin{aligned}
\omega_{0} & :=1 \\
\omega_{1} & :=\alpha_{1} \sqrt{m_{1}} e_{1} \\
\vdots & \vdots \\
\omega_{k} & :=\alpha_{k} \sqrt{m_{k}} e_{k} \\
\omega_{1} \cdot \omega_{2} & :=\alpha_{1} \alpha_{2} \sqrt{m_{1} m_{2}} e_{1} e_{2} \\
\vdots & \vdots \\
\left(\left(\omega_{1} \cdots\right) \omega_{k-1}\right) \cdot \omega_{k} & :=\alpha_{1} \cdots \alpha_{k} \sqrt{m_{1} \cdots m_{k}}\left(\left(e_{1} \cdots\right) e_{k-1}\right) \cdot e_{k}
\end{aligned}
$$


and where one chooses all the appearing components $\alpha_{j_{1} \cdots j_{r}}$ to be rational numbers. An arbitrary $\mathbb{Z}$ linear combination of these lattice elements then has the form

$$
\begin{gathered}
\gamma_{0}+\gamma_{1} \omega_{1}+\cdots+\gamma_{k} \omega_{k}+\cdots+\gamma_{1 \cdots k}\left(\omega_{1}(\cdots)\right) \omega_{k} \\
=\gamma_{0}+\gamma_{1} \alpha_{1} \sqrt{m_{1}} e_{1}+\cdots+\gamma_{k} \alpha_{k} \sqrt{m_{k}} e_{k}+\cdots \\
\quad+\gamma_{1 \cdots k} \alpha_{1 \cdots k} \sqrt{m_{1} \cdots m_{k}}\left(\left(e_{1} \cdots\right) e_{k-1}\right) e_{k},
\end{gathered}
$$

where all $\gamma_{j_{1} \cdots j_{r}}$ are integers. As one easily may verify, the product of such two elements again gives an element of the same form, for instance,

$$
\omega_{1} \cdot \omega_{2}=\alpha_{1} \alpha_{2} \sqrt{m_{1} m_{2}} e_{1} e_{2} .
$$

Note that in $\mathcal{C}_{k}$ one has that $\left(e_{j} e_{k}\right) e_{m}= \pm\left(e_{m} e_{j}\right) e_{k}$ which, however, means that the second structure constants are not anti-symmetric, in general. In the case where all elements $\alpha_{j_{1} \cdots j_{r}}$ are integers, then one easily gets lattices being even closed under multiplication. One can directly observe that these lattices all form rational (resp. integral) Brandt algebras in the Cayley-Dickson algebra and that they are stable under conjugation. The components of the primitive lattice generators are elements from the multiquadratic number field $\mathbb{Q}\left[\sqrt{m_{1}}, \ldots, \sqrt{m_{k}}\right]$. In the particular complex case we are dealing with the classical CM-lattices of the form $\mathbb{Z}+\mathbb{Z} \tau$ where $\tau \in$ $\mathbb{Q}\left[e_{1} \sqrt{m_{1}}\right]$. In the octonionic case we deal with tri-quadratic number fields. Slightly more generally, consider eight $\mathbb{R}$ linearly independent octonionic lattice generators $\omega_{h}(h=0, \ldots, 7)$ where

$$
\begin{aligned}
\omega_{h}= & \alpha_{h_{0}}+\alpha_{h_{1}} \sqrt{m_{1}} e_{1}+\alpha_{h_{2}} \sqrt{m_{2}} e_{2}+\alpha_{h_{3}} \sqrt{m_{3}} e_{3} \\
& +\alpha_{h_{4}} \sqrt{m_{1} m_{2}} e_{4}+\alpha_{h_{5}} \sqrt{m_{1} m_{3}} e_{5}+\alpha_{h_{6}} \sqrt{m_{2} m_{3}} e_{6} \\
& +\alpha_{h_{7}} \sqrt{m_{1} m_{2} m_{3}} e_{7}, \quad \alpha_{h_{j}} \in \mathbb{Q} .
\end{aligned}
$$

It is easy to check that any product $\omega_{h} \omega_{l}$ turns out to be of the same form.

For the sake of completeness, we introduce the notation $W=\left(\omega_{h l}\right)_{h l}(h, l \in$ $\left.\left\{0, \ldots, 2^{k}-1\right\}\right)$ for the matrix of the components of the lattice generators $\omega_{h}$ represented in the basis $\omega_{h}=\omega_{h, 0}+\omega_{h, 1} e_{1}+\omega_{h, 2} e_{2}+\cdots+\omega_{h, 2^{k}-1}\left(e_{1}(\cdots) e_{k-1}\right) e_{k}$. Furthermore, $\operatorname{det}(W)$ stands for its determinant and $\theta_{h, j}$ represents the adjoint determinant, also called co-factor, associated with the element $\omega_{h, j}$.

\section{Algebraic relations between the CM-division values of octonionic regular elliptic functions}

\subsection{Cayley-Dickson regular functions and their basic properties}

To make the paper self-contained, we briefly summarize the basic facts on CayleyDickson regular functions in the sense of the Riemann approach and, in particular, on octonionic regular (monogenic) functions that are needed to prove the main results of this paper. Apart from this regularity concept there is also the concept of sliceregularity in these algebras. See, for instance, [11] and the very recent paper [17] which is based on a different geometric splitting. However, in this paper we restrict ourselves to focus entirely on the following definition. 
Definition 4.1 (Cayley-Dickson regularity (cf. $[7,16])$ ). Let $U$ be an open subset in the Cayley-Dickson algebra $\mathcal{C}_{k}$. A function $f: U \rightarrow \mathcal{C}_{k}$ is called left (right) CayleyDickson regular if $\mathcal{D} f(z)=0$ (resp. $f(z) \mathcal{D}=0$ ) for all $z \in U$, where

$$
\mathcal{D}:=\frac{\partial}{\partial x_{0}}+\sum_{j=1}^{2^{k}-1} \frac{\partial}{\partial x_{j}} e_{j}
$$

is the generalized Cauchy-Riemann operator in the Cayley-Dickson algebra $\mathcal{C}_{k}$.

In the case $k=3$ we get the class of octonionic monogenic functions, discussed in $[6,13,25,26,30,31,32,33]$, which will be called $\mathbb{O}$-regular functions for short in all that follows. If $k=4$, then we deal with the sedenionic monogenic functions, see also [16]. The general case has been addressed in [7].

All left and right Cayley-Dickson regular functions are also harmonic. They satisfy $\sum_{j=0}^{2^{k}-1} \frac{\partial^{2} f}{\partial x_{i}^{2}}=0$.

As important example of a function that is left and right Cayley-Dickson regular serves the generalized Cauchy kernel function $q_{0}(z):=\frac{\bar{z}}{|z|^{2^{k}}}$. Precisely speaking, it is left and right regular at any point $z \neq 0$. In the octonionic case one has $q_{\mathbf{0}}(z)=\frac{\bar{z}}{|z|^{8}}$. As a direct consequence, also all partial derivatives

$$
q_{\mathbf{n}}(z):=\frac{\partial^{|\mathbf{n}|}}{\partial x_{1}^{n_{1}} \cdots \partial x_{2^{k}-1}^{n_{2^{k}-1}}} q_{\mathbf{0}}(z), \quad \mathbf{n}:=\left(n_{1}, \ldots, n_{2^{k}-1}\right),|\mathbf{n}|=\sum_{j=1}^{2^{k}-1} n_{j},
$$

are left and right $\mathcal{C}_{k}$-regular at all points with $z \neq 0$.

Following K. Imaeda, in the algebras $\mathcal{C}_{k}$ up from $k>3$ one cannot set up a general Cauchy integral formula with this kernel function anymore, because the second structure constants are not anti-symmetric in these cases, cf. [16]. This represents a serious obstacle. This might be a reason why so far there has not been spent that much effort to develop a comprehensive generalized function theory in the algebras $\mathcal{C}_{k}$ with $k>3$.

However, in the octonionic case, one still gets a Cauchy integral formula. Nevertheless, notice that in contrast to the Clifford analysis setting, one has to be careful with how to bracket the expressions together. From [32] and elsewhere we may recall:

Theorem 4.2 (Octonionic Cauchy-integral formula). Let $U \subseteq \mathbb{O}$ be open and $K \subset U$ be the closure of an open domain in $(\mathbb{O}$ with an orientable strongly Lipschitz boundary $\partial K$. Let $f: U \rightarrow \mathbb{O}$ be a left (right) $\mathbb{O}$-regular function. Then, under the condition that $z \in \operatorname{int}(K)$ we have

$$
f(z)=\frac{1}{\omega_{8}} \int_{\partial K} q_{0}(w-z) \cdot(d \sigma(w) f(w))
$$


respectively,

$$
f(z)=\frac{1}{\omega_{8}} \int_{\partial K}(f(w) d \sigma(w)) \cdot q_{0}(w-z),
$$

where $\omega_{8}=\frac{\pi^{4}}{3}$ is the surface measure of the unit hypersphere in $\mathbb{O}$. Here $d \sigma(w)=$ $\sum_{i=0}^{7}(-1)^{i} e_{i} \hat{d w_{i}}$ is the oriented octonionic surface differential form where $\hat{d w_{i}}=d w_{0} \wedge$ $d w_{1} \wedge \cdots d w_{i-1} \wedge d w_{i+1} \cdots \wedge d w_{7}$.

Following [16], even in the non-alternative cases, every function $f: U \rightarrow \mathcal{C}_{k}$ that is left (right) $\mathcal{C}_{k}$-regular in an open neighborhood around a point $a \in U$ can locally be expanded in a Taylor series of the form

$$
f(z)=\sum_{|\mathbf{n}|=0}^{+\infty} V_{\mathbf{n}}(z-a) a_{\mathbf{n}}, \quad \text { resp. } \quad f(z)=\sum_{|\mathbf{n}|=0}^{+\infty} a_{\mathbf{n}} V_{\mathbf{n}}(z-a),
$$

where $a_{\mathbf{n}}:=\frac{\partial^{|\mathbf{n}|}}{\partial \mathbf{x}^{\mathbf{n}}} f(a)$ are hypercomplex numbers from $\mathcal{C}_{k}$ and the polynomials $V_{\mathbf{n}}(z)$ are the generalized Fueter polynomials. This is a consequence of the power associativity that remains valid in all Cayley-Dickson algebras.

In the Cayley-Dickson algebra setting, the Fueter polynomials have the form

$$
V_{\mathbf{n}}(z)=\frac{1}{|\mathbf{n}| !} \sum_{\pi \in \operatorname{perm}(\mathbf{n})}\left(Z_{\pi\left(n_{1}\right)}\left(Z_{\pi\left(n_{2}\right)}\left(\cdots\left(Z_{\pi\left(n_{2^{k}-2}\right)} Z_{\pi\left(n_{2^{k}-1}\right)}\right) \cdots\right)\right)\right) .
$$

Here, $\operatorname{perm}(\mathbf{n})$ denotes the set of all distinguishable permutations of the sequence $\left(n_{1}, n_{2}, \ldots, n_{2^{k}-1}\right)$ and $Z_{i}:=V_{\tau(i)}(z):=x_{i}-x_{0} e_{i}$ for all $i=1, \ldots, 2^{k}-1$, see $[31$, Theorem C, p. 208], where the octonionic case has been treated specifically.

As in the complex and Clifford analysis setting, Cauchy's integral formula allows us easily to show the particular octonionic case the following.

Theorem 4.3 (Octonionic Liouville theorem). If $f: \mathbb{O} \rightarrow \mathbb{O}$ is left or right $\mathbb{O}$-regular and bounded over the whole algebra $\mathbb{O}$, then $f$ must be a constant.

Proof. We describe the left $\mathbb{O}$-regular case. By performing partial differentiation on the octonionic Cauchy integral formula, one directly obtains that

$$
\frac{\partial}{\partial x_{i}} f(z)=\frac{1}{\omega_{8}} \int_{|z-w|=r} q_{\tau(i)}(w-z) \cdot(d \sigma(w) \cdot f(w)) .
$$

Thus, in view of $\left|q_{\tau(i)}(z-w)\right| \leq M|z-w|^{-8}$ (with a real constant $M$ ) and since the measure of the surface of a 8 -dimensional ball of radius $r$ is $\frac{\pi^{4} r^{7}}{3}$, we have

$$
\left|\frac{\partial}{\partial x_{i}} f(z)\right| \leq \frac{M}{r} \sup _{z \in \mathbb{O}}\{|f(z)|\}
$$

which tends to zero, because $\sup _{z \in \mathbb{O}}\{|f(z)|\}$ is bounded. Hence, $f$ must be constant. 
Note that we have used the Cauchy integral formula which is not available for higher-dimensional Cayley-Dickson algebras. Therefore, this simple proof cannot be extended directly to the more general algebras $\mathcal{C}_{k}$ for $k>3$.

More generally, on can also establish that even every function $f: \mathbb{O} \rightarrow \mathbb{O}$ that is harmonic and bounded over the whole algebra $\mathbb{O}$ is a constant. This actually can be done by classical harmonic analysis.

In particular, for the octonionic case one may also introduce in view of the property of being a composition algebra:

Definition 4.4 (Octonionic meromorphicity). Let $U \subseteq \mathbb{O}$ be an open set. Suppose that $a \in U$ and that $f: U \backslash\{a\} \rightarrow \mathbb{O}$ is left (right) $\mathbb{O}$-regular. Then the point $a$ is called a non-essential isolated singularity of $f$ if there exists a non-negative integer $n$ such that $|z-a|^{n}|f(z)|$ remains bounded in a neighborhood around $a$. Left (right) (O-regular functions that have at most unessential singular points in a discrete subset $S \subset \mathbb{O}$ are called left (right) $\mathbb{O}$-meromorphic.

More generally, one can also extend the notion of octonionic meromorphicity to functions which have non-essential singularities on manifolds with boundary, namely, by defining non-essential singular sets $S$ in the same way as W. Nef did for the quaternionic case in $[24,10]$. This is technically more complicated. For simplicity we therefore restrict ourselves to only address octonionic functions with isolated singularities in this paper, although a more general treatment is possible.

\subsection{Basic properties of octonionic regular elliptic functions}

In this subsection we briefly summarize the most basic properties of octonionic regular elliptic functions. We start by giving its definition which is similar to that addressing the quaternionic case in [10] and to that addressing paravector-valued functions with values in associative Clifford algebras in [21].

Definition 4.5. Let $\Omega_{8}=\mathbb{Z} \omega_{0}+\ldots+\mathbb{Z} \omega_{7}$ be an arbitrary eight-dimensional octonionic lattice; that means that $\omega_{0}, \ldots, \omega_{7}$ are supposed to be eight $\mathbb{R}$-linearly independent octonions. Further, let $S \subset \mathbb{O}$ be a discrete subset. A left (right) $\mathbb{O}$-regular function $f: \mathbb{O} \backslash S \rightarrow \mathbb{O}$ that has atmost unessential singularities at the points of $S$ is called a left (right) $\mathbb{O}$-regular elliptic function if it satisfies at each $z \in \mathbb{O} \backslash S$ that $f(z+\omega)=$ $f(z)$ for all $\omega \in \Omega_{8}$ and $S+\omega=S$ for all $\omega \in \Omega_{8}$.

Remark 4.6. A crucial difference to the Clifford analysis setting consists in the fact that the set of left (right) (O)-regular elliptic functions is not a right (left) nonassociative $\mathbb{O}$-module, see [19] Remark 4.4. But one still has the property that left (right) $\mathbb{O}$-regularity is inherited by partial derivation of such a function.

Like in complex and Clifford analysis (cf. [21, 27]) it is not possible to find any non-constant $\Omega_{8}$-periodic function that is left or right $\mathbb{O}$-regular on the whole algebra (O). This is due to the fact that the topological quotient $\mathbb{O} / \Omega_{8}$ is a compact 8 -torus. However, any function that is $\mathbb{O}$-regular on the whole algebra $\mathbb{O}$ is, in paticular, continuous. Hence, it is bounded on the topological quotient torus, which means 
that it is bounded on the closure of each period cell. Therefore, as a consequence of the generalized octonionic Liouville theorem, such a function must be a constant. The same holds under the weaker condition of being harmonic.

Therefore, a non-constant $\mathbb{O}$-regular or harmonic $\Omega_{8}$-periodic function must have singularities.

Examples. The simplest non-trivial examples of $\mathbb{O}$-regular functions are given in terms of the $\Omega_{8}$-periodization of the partial derivatives of the octonionic Cauchy kernel function $q_{\mathbf{0}}(z)$. Applying the classical Eisenstein series convergence argument, the series

$$
\wp_{\mathbf{n}}(z):=\sum_{\omega \in \Omega_{8}}\left[\left(\frac{\partial^{\mid \mathbf{n}} \mid}{\partial \mathbf{x}^{\mathbf{n}}} q_{\mathbf{0}}\right)(z+\omega)\right]
$$

converges normally whenever $|\mathbf{n}|:=\sum_{j=1}^{7} n_{j} \geq 2$, since $\sum_{\omega \in \Omega_{8} \backslash\{0\}}|\omega|^{-(7+\alpha)}$ is convergent if and only if $\alpha>1$. In the limit case where $\mathbf{n}=\tau(i)$ is a multi-index of length 1 (where $n_{j}=\delta_{i j}$ for one particular $i \in\{1, \ldots, 7\}$ ) the series $\sum_{\omega \in \Omega_{8}} q_{\tau(i)}(z+\omega)$ where $q_{\tau(i)}(z)=\frac{\partial}{\partial x_{i}} q_{\mathbf{0}}(z)$ is not convergent anymore. However, as usual, convergence can be achieved by adding a convergence-preserving term in the way

$$
\wp_{\tau(i)}(z)=q_{\tau(i)}(z)+\sum_{\omega \in \Omega_{8} \backslash\{0\}}\left(q_{\tau(i)}(z+\omega)-q_{\tau(i)}(\omega)\right),
$$

similar to the Clifford algebra case, cf. [10, 21, 27].

In fact, in complete analogy to the Clifford analysis setting one can prove:

Proposition 4.7. The series $\wp_{\tau(i)}(z)$ are $\Omega_{8}$-periodic.

Proof. To prove the periodicity, one can apply the same chain of arguments as in the complex case. From a direct rearrangement argument it is clear that for $|\mathbf{n}| \geq 2$ the series $\wp_{\mathbf{n}}$ satisfy $\wp_{\mathbf{n}}(z+\omega)=\wp_{\mathbf{n}}(z)$ for all $z \in \mathbb{O} \backslash \Omega_{8}$ and all $\omega \in \Omega_{8}$. So, for any $i$, the difference of $\wp_{\tau(i)}(z+\omega)-\wp_{\tau(i)}(z)$ must be a constant, say $C$. Let $\omega_{h}$ be a primitive period of $\Omega_{8}$ and consider $z=-\frac{\omega_{h}}{2}$. Furthermore, note that $\wp_{\tau(i)}$ is an even function, because each term in the series representation is even. $q_{\tau(i)}$ is even, because it is the partial derivative of $q_{\mathbf{0}}$, which obviously is odd. Therefore, the difference $\wp_{\tau(i)}(z+\omega)-\wp_{\tau(i)}(z)$ becomes $\wp_{\tau(i)}\left(-\frac{\omega_{h}}{2}+\omega\right)-\wp_{\tau(i)}\left(-\left(-\frac{\omega_{h}}{2}\right)\right)=$ $\wp_{\tau(i)}\left(\frac{\omega_{h}}{2}\right)-\wp_{\tau(i)}\left(\frac{\omega_{h}}{2}\right)=0$, thus $C=0$ and the $\Omega_{8}$ periodicity is established, since $\omega_{h}$ was an arbitrarily chosen primitive period.

Remark 4.8. If we consider the subseries that arises by summing only over the lattice points of a seven-dimensional sublattice $\Omega_{7}$ whose elements $\omega \in \Omega_{7}$ satisfy all $\mathcal{S}(\omega)=0$, where $\mathcal{S}(\omega)=\omega+\bar{\omega}$ means the trace as defined in Definition 3.1, then we deal with a partial derivative of the octonionic generalized cotangent function from $[25]$. 
The left $\mathbb{O}$-regular primitive of the fully $\Omega_{8}$-periodic function $\wp_{\tau(i)}$ given by

$$
\zeta(z):=q_{\mathbf{0}}(z)+\sum_{\omega \in \Omega_{8} \backslash\{0\}}\left(q_{\mathbf{0}}(z+\omega)-q_{\mathbf{0}}(\omega)+\sum_{j=1}^{7} V_{\tau(j)}(z) q_{\tau(j)}(\omega)\right)
$$

provides us with the direct analogue of the Weierstraß $\zeta$-function, satisfying, like in the Clifford case, $\frac{\partial \zeta}{\partial x_{i}}=\wp_{\tau(i)} . \zeta(z)$ is not $\Omega_{8}$-periodic anymore. However, $\zeta(z)$ is quasi $\Omega_{8}$-periodic, which means that

$$
\zeta\left(z+\omega_{h}\right)-\zeta(z)=\eta_{h}
$$

where $\eta_{h}$ are octonionic constants, the so-called octononic Legendre constants. In the latter equation $\omega_{h}(h=0, \ldots, 7)$ represent the primitive periods of $\Omega_{8}$. The Legendre constants are given by

$$
\eta_{h}=\zeta\left(-\frac{\omega_{h}}{2}+\omega_{h}\right)-\zeta\left(-\frac{\omega_{h}}{2}\right)=2 \zeta\left(\frac{\omega_{h}}{2}\right),
$$

because $\zeta$ is an odd function which can readily be seen by a rearrangement of the series.

Remark 4.9. The left $\mathbb{O}$-regularity of $\zeta(z)$ follows from the application of the Weierstraß convergence theorem from [32, Theorem 11] to each particular term of the series. Note that

$$
\begin{aligned}
\mathcal{D}\left[V_{\left.\tau(j)(z) q_{\tau(j)}(\omega)\right]}=\right. & \frac{\partial}{\partial x_{0}}\left(x_{j}-x_{0} e_{j}\right) q_{\tau(j)}(\omega) \\
& +e_{j}\left[\frac{\partial}{\partial x_{j}}\left(x_{j}-x_{0} e_{j}\right) q_{\tau(j)}(\omega)\right] \\
& +\sum_{i \neq 0, j} e_{j}\left[\frac{\partial}{\partial x_{i}}\left(x_{j}-x_{0} e_{j}\right) q_{\tau(j)}(\omega)\right] \\
= & -e_{j} q_{\tau(j)}(\omega)+e_{j} q_{\tau(j)}(\omega)=0 .
\end{aligned}
$$

The left and right $\mathbb{O}$-regularity of the functions $\wp_{\tau(i)}$ and $\wp_{\mathbf{n}}$ with $|\mathbf{n}| \geq 2$ is evident.

The detailed proof of convergence follows along the same lines as in the Clifford case. Hence, we omit it. See [21] and see also [25] for the particular octonionic cotangent series constructions treated there.

Remark 4.10. The same series constructions can also be made in the general $2^{k}$ dimensional Cayley-Dickson algebras by inserting the functions $q_{\mathbf{0}}(z):=\frac{\bar{z}}{|z|^{2^{k}}}$ or their partial derivatives, respectively, in the series constructions. The convergence conditions remain the same. However, notice that we do not have an analogue of Liouville's theorem in the more general setting due to the lack of a direct analogue of Cauchy's integral formula. Anyway, the Taylor expansion representation of the Cauchy kernel function and its partial derivatives which are required in the convergence proof remain valid. 


\subsection{Octonionic regular elliptic functions for generalized CM-lattices and their division values}

The results presented in this subsection only address the octonionic setting, because the proofs explicitly use the alternative property of the octonions which is lost in the higher-dimensional Cayley-Dickson algebras $\mathcal{C}_{k}$ with $k>3$.

Before we start, we need to establish some important preparatory statements.

First we note that

$$
\begin{aligned}
q_{0}((\lambda z) \cdot \mu) & =\frac{\overline{(\lambda z) \cdot \mu}}{|(\lambda z) \cdot \mu|^{8}}=\frac{\bar{\mu} \cdot(\overline{\lambda z})}{|\mu|^{8}|\lambda z|^{8}} \\
& =\frac{\bar{\mu} \cdot(\bar{z} \bar{\lambda})}{|\mu|^{8}|z|^{8}|\lambda|^{8}}=q_{\mathbf{0}}(\mu) \cdot\left(q_{\mathbf{0}}(z) q_{\mathbf{0}}(\lambda)\right) .
\end{aligned}
$$

Analogously, one obtains

$$
\begin{aligned}
q_{\mathbf{0}}(\lambda \cdot(z \mu)) & =\frac{\overline{\lambda \cdot(z \mu)}}{|\lambda \cdot(z \mu)|^{8}}=\frac{\overline{z \mu} \cdot \bar{\lambda}}{|\lambda|^{8}|z \mu|^{8}} \\
& =\frac{(\bar{\mu} \bar{z}) \cdot \bar{\lambda}}{|\mu|^{8}|z|^{8}|\lambda|^{8}}=\left(q_{\mathbf{0}}(\mu) q_{\mathbf{0}}(z)\right) \cdot q_{\mathbf{0}}(\lambda)
\end{aligned}
$$

Here we used the conjugation property $\overline{a b}=\bar{b} \bar{a}$.

Now we want to show the following formula.

Proposition 4.11. For each $\lambda, \mu, z \in \mathbb{O} \backslash\{0\}$ we have

$$
\left(\mu \cdot q_{0}((\lambda z) \cdot \mu)\right) \cdot \lambda=\frac{1}{(\mathcal{N}(\mu) \mathcal{N}(\lambda))^{3}} q_{0}(z)
$$

Proof. Here, we have to argue very carefully, since we do not have associativity. So, we do it step by step. First we note that

$$
\mu \cdot\left(q_{\mathbf{0}}(z \mu)\right)=\mu \cdot\left(\frac{\overline{z \mu}}{|z \mu|^{8}}\right)=\mu \cdot\left(\frac{\bar{\mu} \bar{z}}{|\mu|^{8}|z|^{8}}\right)=\frac{(\mu \bar{\mu}) \bar{z}}{|\mu|^{8}|z|^{8}}=\frac{1}{|\mu|^{6}} q_{\mathbf{0}}(z)
$$

where we exploited the alternating property $\mu(\bar{\mu} \bar{z})=(\mu \bar{\mu})(\bar{z})$. Here, we exploited a special property of the octonions that cannot be extended to sedenions or the following Cayley-Dickson algebras. 
Next we put $f(z):=\mu \cdot\left(q_{\mathbf{0}}(z \mu)\right)=\frac{1}{|\mu|^{6}} q_{\mathbf{0}}(z)$. And now we can conclude that

$$
\begin{aligned}
\left.\left(\mu \cdot q_{0}((\lambda z) \cdot \mu)\right)\right) \cdot \lambda & =f(\lambda z) \cdot \lambda \\
& =\frac{1}{|\mu|^{6}} q_{0}(\lambda z) \cdot \lambda \\
& =\frac{1}{|\mu|^{6}}\left(\frac{\overline{\lambda z}}{|\lambda z|^{8}}\right) \cdot \lambda \\
& =\frac{(\bar{z} \bar{\lambda}) \cdot \lambda}{|\mu|^{6}|z|^{8}|\lambda|^{8}}=\frac{\bar{z} \cdot(\bar{\lambda} \cdot \lambda)}{|\mu|^{6}|z|^{8}|\lambda|^{8}} \\
& =\frac{1}{|\mu|^{6}} \frac{1}{|\lambda|^{6}} q_{\mathbf{0}}(z)=\frac{1}{(\mathcal{N}(\mu) \mathcal{N}(\lambda))^{3}} q_{\mathbf{0}}(z) .
\end{aligned}
$$

Remark 4.12. We wish to emphasize clearly that this formula holds for octonions. Our argumentation cannot be extended beyond octonions in the next steps of the usual Cayley-Dickson doubling, at least not by using this chain of arguments, because we explicitly used the alternative property.

As a direct consequence of the fact that $\frac{1}{(\mathcal{N}(\mu) \mathcal{N}(\lambda))^{3}}$ is real-valued we obtain the following important statement.

Proposition 4.13. For all $\lambda, \mu \in \mathbb{O} \backslash\{0\}$ the functions $\left.\left(\mu \cdot q_{0}((\lambda z) \cdot \mu)\right)\right) \cdot \lambda$ and, in particular, $q_{\mathbf{0}}(\lambda z) \cdot \lambda$ and $\mu \cdot q_{\mathbf{0}}(z \mu)$ are left and right $\mathbb{O}$-regular at each $z \in \mathbb{O} \backslash\{0\}$.

Remark 4.14. Note again that in contrast to the Clifford analysis setting the property of the latter proposition is not immediate, because left (right) $\mathbb{O}$-regular functions do not form a right (left) $\mathbb{O}$-module as mentioned before. The property is true for the particular function $q_{\mathbf{0}}$ but at least as far as we know it is not evident for any arbitrary left (right) $\mathbb{O}$-regular function $f$.

Now let particularly $\Omega_{8}=\mathbb{Z}+\mathbb{Z} \omega_{1}+\cdots+\mathbb{Z} \omega_{7}\left(\omega_{0}=1\right)$ be a lattice with octonionic multiplication as defined in Section 3. Suppose that $\lambda \in \mathbb{O} \backslash \mathbb{R}$ is a nontrivial multiplicator from a left ideal $\mathcal{L}$ with $\mathcal{L} \Omega_{8} \subseteq \Omega_{8}$ and assume that $\mu \in \mathbb{O} \backslash \mathbb{R}$ is a non-trivial multiplicator from a right ideal $\mathcal{R}$ with $\Omega_{8} \mathcal{R} \subseteq \Omega_{8}$.

We look at the associated octonionic left $\mathbb{O}$-regular Weierstraß $\zeta$-function

$$
\zeta(z)=\zeta(z, \Omega)=q_{\mathbf{0}}(z)+\sum_{\omega \in \Omega_{8} \backslash\{0\}}\left[q_{\mathbf{0}}(z+\omega)-q_{\mathbf{0}}(\omega)+\sum_{j=1}^{7} V_{\tau(j)}(z)\left(q_{\tau(j)}(\omega)\right)\right] .
$$

If $\Omega_{8}$ is such a lattice and $\mathcal{L}$ is such a left ideal, then we can find a $\lambda \in \mathcal{L} \backslash \mathbb{R}$ such that $\lambda \omega \in \Omega_{8}$ for all $\omega \in \Omega_{8}$.

So, the function $\zeta(\lambda z) \cdot \lambda$ is a well-defined quasi-elliptic function on the same lattice $\Omega_{8}$, since $\lambda \omega \in \Omega_{8}$ for all $\omega \in \Omega_{8}$. Note that quasi-ellipticity means that the function is $\Omega_{8}$-periodic up to a constant. 
It is easy to see that it is at least harmonic when applying the Weierstraß convergence theorem to each term of the series. According to Proposition 4.13 the term $q_{\mathbf{0}}(\lambda z) \lambda$ is left and right $\mathbb{O}$-regular and hence harmonic. The terms $V_{\tau(j)}(z)\left(q_{\tau(j)}(\omega)\right)$ are all linear and therefore in the kernel of the Laplacian. Since the Laplacian is a scalar operator, one has

$$
\Delta\left[q_{0}(\lambda z+\omega) \lambda\right]=\Delta\left[q_{0}(\lambda z+\omega)\right] \lambda .
$$

So, since $0=\Delta\left[q_{0}(\lambda z) \lambda\right]$ and since $\mathbb{O}$ is a division algebra, one also has $\Delta\left[q_{0}(\lambda z)\right]=$ 0 . Since $\omega \neq 0$, one can find a $t \in \mathbb{O}$ such that $\omega=\lambda t$. Applying a linear shift in the argument also leads to the fact that

$$
\begin{aligned}
\Delta\left[q_{\mathbf{0}}(\lambda z+\omega)\right] & =\Delta\left[q_{\mathbf{0}}(\lambda z+\lambda t)\right]=\Delta\left[q_{\mathbf{0}}(\lambda(z+t))\right] \\
& =\Delta\left[\left(q_{\mathbf{0}}(z+t)\right) \frac{\bar{\lambda}}{|\lambda|^{8}}\right]=\Delta\left[\left(q_{\mathbf{0}}(z+t)\right)\right] \frac{\bar{\lambda}}{|\lambda|^{8}}=0,
\end{aligned}
$$

since the differential remains invariant under the shift $z+t$.

Analogously, there are elements $\mu \in \mathcal{R} \backslash \mathbb{R}$ such that $\omega \mu \in \Omega_{8}$ for all $\omega \in \Omega_{8}$, so that the function $\mu \cdot \zeta(z \mu)$ is also a well-defined quasi-elliptic function again on the same lattice, since also $\omega \mu \in \Omega_{8}$ for all $\omega \in \Omega_{8}$. Here again, we can establish that this function is at least harmonic.

More generally, and bearing in mind the non-associativity, the two functions

$$
\zeta_{\lambda, \mu}^{1}(z):=(\mu \cdot \zeta((\lambda z) \cdot \mu)) \cdot \lambda
$$

and

$$
\zeta_{\lambda, \mu}^{2}(z):=\mu \cdot(\zeta(\lambda \cdot(z \mu)) \cdot \lambda)
$$

are well-defined at least harmonic quasi-elliptic functions for all $\lambda \in \mathcal{L}, \mu \in \mathcal{R}$, since

$$
\left(\lambda \Omega_{8}\right) \cdot \mu \subseteq \Omega_{8} \mu \subseteq \Omega_{8}
$$

and

$$
\lambda \cdot\left(\Omega_{8} \mu\right) \subseteq \lambda \Omega_{8} \subseteq \Omega_{8}
$$

These inclusions are true, because $\Omega_{8}$ is a lattice with Cayley-Dickson multiplication (CM-property).

For the sake of clarity, the quasi-periodicity (periodicity up to a constant) follows explicitly by the calculations

$$
\begin{aligned}
\zeta_{\lambda, \mu}^{1}(z+\omega) & =(\mu \cdot \zeta((\lambda[z+\omega]) \cdot \mu)) \cdot \lambda \\
& =(\mu \cdot \zeta((\lambda z) \cdot \mu+(\lambda \omega) \cdot \mu)) \cdot \lambda \\
& =\left(\mu \cdot \zeta\left((\lambda z) \cdot \mu+\omega^{*}\right)\right) \cdot \lambda \\
& =\left(\mu \cdot \zeta((\lambda z) \cdot \mu)+C\left(w^{*}\right)\right) \cdot \lambda \\
& =(\mu \cdot \zeta((\lambda z) \cdot \mu)) \cdot \lambda+\left(\mu \cdot C\left(\omega^{*}\right)\right) \cdot \lambda \\
& =\zeta_{\lambda, \mu}^{1}(z+\omega)+\tilde{C}\left(\omega^{*}\right),
\end{aligned}
$$


where $C\left(\omega^{*}\right)$ and $\tilde{C}\left(\omega^{*}\right)$ are two constants depending on $\omega^{*}$, which is defined by $\omega^{*}:=(\lambda \omega) \cdot \mu$, which is an element from $\Omega_{8}$, because of the CM-property.

Note that both functions $\zeta_{\lambda, \mu}^{1}(z)$ and $\zeta_{\lambda, \mu}^{2}(z)$, in general, differ from each other as a consequence of the lack of associativity. For the sake of convenience we focus from now on the function $\zeta_{\lambda, \mu}(z):=\zeta_{\lambda, \mu}^{1}(z)$, since the other version can be treated analogously.

The function $(\mu \cdot \zeta((\lambda z) \cdot \mu)) \cdot \lambda$ is singular if and only if $(\lambda z) \cdot \mu=\omega$ for a lattice point $\omega \in \Omega_{8}$. This is equivalent to $(\lambda z)=\omega \mu^{-1} \Longleftrightarrow z=\lambda^{-1} \cdot\left(\omega \mu^{-1}\right)$. Thus, the function $\zeta_{\lambda, \mu}^{1}(z)$ has isolated point singularities at exactly the points $z=\lambda^{-1} \cdot\left(\omega \mu^{-1}\right)$ where $\omega$ runs through $\Omega_{8}$.

By a counting argument, we obtain that $\zeta_{\lambda, \mu}$ has exactly $\mathcal{N}(\lambda \mu)^{4}$-many isolated point singularities in the fundamental periodic cell

$$
\mathcal{F}:=\left\{x=\alpha_{0}+\alpha_{1} \omega_{1}+\cdots+\alpha_{7} \omega_{7} \mid 0 \leq \alpha_{j}<1, j \in\{0, \ldots, 7\}\right\},
$$

which, in particular, contains 0 .

The set of all these singularities that lie in the fundamental set will be denoted by $\mathcal{V}_{\lambda, \mu ; \Omega_{8}}$ in all that follows.

Remark 4.15. In the simple case where $\lambda=2$ and $\mu=1$, this set consists exactly of those points where the coordinates either have the value zero or $1 / 2$, whose cardinality evidently equals $2^{8}=\mathcal{N}(2)^{4}$.

Now we have all pre-requisites to formulate and prove our main theorem.

Theorem 4.16. Let $\Omega_{8}=\mathbb{Z}+\mathbb{Z} \omega_{1}+\cdots+\mathbb{Z} \omega_{7},\left(\omega_{0}:=1\right)$ be an octonionic lattice with octonionic multiplication with the properties and notations as described previuously. The trace of the octonionic division values of the $\mathbb{O}$-regular elliptic functions $\wp_{\tau(i)}$ $(i=1, \ldots, 7)$ can be expressed by

$$
\begin{aligned}
& \sum_{v \in \mathcal{V}_{\lambda, \mu ; \Omega_{8}} \backslash\{0\}} \wp_{\tau(i)}(v) \\
= & -\frac{(\mathcal{N}(\lambda) \mathcal{N}(\mu))^{3}}{\operatorname{det}(W)}\left[\sum_{h=0}^{7} \Theta_{h_{i}}\left(\mu \cdot\left(\sum_{j=0}^{7} n_{h_{j}} \eta_{j}\right)\right) \cdot \lambda-\mathcal{N}(\lambda) \mathcal{N}(\mu) \sum_{h=0}^{7} \Theta_{h_{i}} \eta_{h}\right],
\end{aligned}
$$

where $\Theta_{h i}$ denotes the adjoint determinant (co-factor) associated with the lattice component $\omega_{\text {hi }}$ and where

$$
\mathcal{V}_{\lambda, \mu ; \Omega_{8}}:=\left\{v \in \mathcal{F} \mid v=\lambda^{-1} \cdot\left(\omega \mu^{-1}\right), \omega \in \Omega_{8}\right\} .
$$

Proof. As a consequence of formula (1), we may infer that the Laurent expansion of the function $\zeta_{\lambda, \mu}$ centered at zero has the form

$$
\zeta_{\lambda, \mu}(z)=(\mu \zeta((\lambda z) \cdot \mu)) \cdot \lambda=\frac{1}{(\mathcal{N}(\mu) \mathcal{N}(\lambda))^{3}} q_{0}(z)+A(z),
$$

where $A$ is a function that is at least harmonic in some neighborhood of 0 . 
Conversely, the function

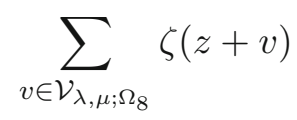

can be written in the form $q_{\mathbf{0}}(z)+B(z)$, where $B(z)$ is also a function that is definetely left $\mathbb{O}$-regular around 0 . Therefore, the difference function

$$
f(z):=\zeta_{\lambda, \mu}(z)-\frac{1}{(\mathcal{N}(\mu) \mathcal{N}(\lambda))^{3}} \cdot \sum_{v \in \mathcal{V}_{\lambda, \mu ; \Omega_{8}}} \zeta(z+v)
$$

is at least harmonic around 0, too. Similarly, one gets the same result if one considers the Laurent expansion around another singular point $v \in \mathcal{V}_{\lambda, \mu ; \Omega_{8}}$.

The same is true for all the partial derivatives $(i \in\{0, \ldots, 7\})$

$$
\begin{aligned}
\frac{\partial}{\partial x_{i}} f(z) & =\frac{\partial}{\partial x_{i}} \zeta_{\lambda, \mu}(z)-\frac{1}{(\mathcal{N}(\mu) \mathcal{N}(\lambda))^{3}} \sum_{v \in \mathcal{V}_{\lambda, \mu ; \Omega_{8}}} \frac{\partial}{\partial x_{i}} \zeta(z+v) \\
& =\frac{\partial}{\partial x_{i}} \zeta_{\lambda, \mu}(z)-\frac{1}{(\mathcal{N}(\mu) \mathcal{N}(\lambda))^{3}} \sum_{v \in \mathcal{V}_{\lambda, \mu ; \Omega_{8}}} \wp_{\tau(i)}(z+v) .
\end{aligned}
$$

The functions $\wp_{\tau(i)}(z+v)$ are all $\Omega_{8}$-periodic, because we proved that $\wp_{i}(z)$ is $\Omega_{8}$ periodic.

The functions $\frac{\partial}{\partial x_{i}} \zeta_{\lambda, \mu}(z)$ are $\Omega_{8}$-periodic, too, because $\zeta_{\lambda, \mu}(z+\omega)-\zeta_{\lambda, \mu}(z)$ turned out to be constant, so the partial derivatives of this expression must be $\Omega_{8}$-periodic.

Consequently, each function $f_{i}:=\frac{\partial f}{\partial x_{i}}$ is $\Omega_{8}$-periodic and must be at least harmonic on the entire algebra $\mathbb{O}$, since it has no singularity inside of $\mathcal{V}_{\lambda, \mu ; \Omega_{8}}$. So, in view of Liouville's theorem there are octonionic constants $C_{i} \in \mathbb{O}$ such that $f_{i}(z)=C_{i}$ for all $z$. Now we make the ansatz

$$
\zeta_{\lambda, \mu}(z)=\frac{1}{(\mathcal{N}(\mu) \mathcal{N}(\lambda))^{3}} \sum_{v \in \mathcal{V}_{\lambda, \mu ; \Omega_{8}}} \zeta(z+v)+\sum_{j=1} V_{\tau(j)}(z) C_{j}+C,
$$

where $C$ is a further octonionic constant.

Now let $\omega_{h}$ be a primitive period of $\Omega_{8}(h=0,1, \ldots, 7)$. Then we have

$$
\begin{aligned}
\zeta_{\lambda, \mu}\left(z+\omega_{h}\right)= & (\mu \cdot \zeta(\lambda(z+\omega) \cdot \mu))) \cdot \lambda \\
= & \frac{1}{(\mathcal{N}(\mu) \mathcal{N}(\lambda))^{3}} \sum_{v \in \mathcal{V}_{\lambda, \mu ; \Omega_{8}}} \zeta\left(z+\omega_{h}+v\right) \\
& +\sum_{j=1}^{7} V_{\tau(j)}\left(z+\omega_{h}\right) C_{j}+C
\end{aligned}
$$

for all $h \in\{0,1, \ldots, 7\}$. 
Now the crucial aspect is that the lattice $\Omega_{8}$ has octonionic multiplication of the form $\left(\lambda \Omega_{8}\right) \mu \subseteq \Omega_{8}$ and $\lambda\left(\Omega_{8} \mu\right) \subseteq \Omega$. Therefore, there exist integers $n_{h_{j}} \in \mathbb{Z}$ such that

$$
\left(\lambda \omega_{h}\right) \cdot \mu=\sum_{j=0}^{7} n_{h_{j}} \omega_{j}
$$

In view of the Legendre relation that we stated in the previous subsection, we have the additive relation

$$
\zeta\left((\lambda z) \cdot \mu+\left(\lambda \omega_{h}\right) \cdot \mu\right)=\zeta((\lambda z) \cdot \mu)+\sum_{j=0}^{7} n_{h_{j}} \eta_{j}
$$

with the octonionic Legendre constants $\eta_{0}, \ldots, \eta_{7}$.

Applying formula (4) we get

$$
\begin{aligned}
& \left(\mu \cdot \zeta\left(\left(\lambda\left(z+\omega_{h}\right)\right) \cdot \mu\right)\right) \cdot \lambda \\
& =\frac{1}{(\mathcal{N}(\mu) \mathcal{N}(\lambda))^{3}} \sum_{v \in \mathcal{V}_{\lambda, \mu ; \Omega_{8}}} \zeta\left(z+\omega_{h}+v\right)+\sum_{j=1}^{7} V_{\tau(j)}\left(z+\omega_{h}\right) C_{j}+C .
\end{aligned}
$$

Using the Legendre relation, the latter equation is equivalent to

$$
\begin{aligned}
& \left(\mu \cdot \zeta\left((\lambda z) \cdot \mu+\left(\lambda \omega_{h}\right) \cdot \mu\right)\right) \cdot \lambda \\
& =\frac{1}{(\mathcal{N}(\mu) \mathcal{N}(\lambda))^{3}} \sum_{v \in \mathcal{V}_{\lambda, \mu ; \Omega_{8}}}\left[\zeta(z+v)+\eta_{h}\right] \\
& \quad+\sum_{j=1}^{7} V_{\tau(j)}\left(z+\omega_{h}\right) C_{j}+C .
\end{aligned}
$$

Applying (6) to the previous equation leads to

$$
\begin{aligned}
& (\mu \cdot \zeta((\lambda z) \cdot \mu)) \cdot \lambda+\left(\mu\left(\sum_{j=0}^{7} n_{h_{j}} \eta_{j}\right)\right) \cdot \lambda \\
& =\frac{1}{(\mathcal{N}(\mu) \mathcal{N}(\lambda))^{3}} \sum_{v \in \mathcal{V}_{\lambda, \mu ; \Omega_{8}}}\left[\zeta(z+v)+\eta_{h}\right]+\sum_{j=1}^{7} V_{\tau(j)}(z) C_{j}+C \\
& \quad+\sum_{j=1}^{7} V_{\tau(j)}\left(\omega_{h}\right) C_{j} .
\end{aligned}
$$

Since the first term of the left-hand side equals the expression of the sum of the first two terms of the right-hand side in view of (4), we obtain the relation

$$
\left(\mu \cdot\left(\sum_{j=0}^{7} n_{h_{j}} \eta_{j}\right)\right) \cdot \lambda=\frac{1}{(\mathcal{N}(\mu) \mathcal{N}(\lambda))^{3}} \sum_{v \in \mathcal{V}_{\lambda, \mu ; \Omega_{8}}} \eta_{h}+\sum_{j=1}^{7} V_{\tau(j)}\left(\omega_{h}\right) C_{j} .
$$


Next, since the cardinality of $\mathcal{V}_{\lambda, \mu ; \Omega_{8}}$ equals $\mathcal{N}(\lambda \mu)^{4}$, we have

$$
\sum_{v \in \mathcal{V}_{\lambda, \mu ; \Omega_{8}}} \eta_{h}=\mathcal{N}(\lambda \mu)^{4} \cdot \eta_{h}
$$

Thus, we arrive at

$$
\left(\mu \cdot\left(\sum_{j=0}^{7} n_{h_{j}} \eta_{j}\right)\right) \cdot \lambda=\mathcal{N}(\lambda \mu) \eta_{h}+\sum_{j=1}^{7}\left(\omega_{h_{j}}-e_{j} \omega_{h_{0}}\right) C_{j}
$$

where we put $\omega_{h}=\sum_{j=0}^{7} \omega_{h_{j}} e_{j}$ for the representation of the primitive periods $\omega_{h}$ in the coordinates of the canonical basis elements $e_{0}, e_{1}, \ldots, e_{7}$.

Next, let us write $\Theta_{h_{j}}$ for the adjoint determinant (co-factor) associated with the element $\omega_{h_{j}}$. Then, classical linear algebra tells us that

$$
\sum_{h=0}^{7} \omega_{h_{i}} \Theta_{h_{l}}=\delta_{i l} \operatorname{det}(W) \text {. }
$$

Note that the elements $\Theta_{h_{l}}$ are all real numbers. Combining the formula (8) with (7), we obtain that

$$
\begin{aligned}
& \sum_{h=0}^{7}\left[\Theta_{h_{i}}\left(\mu \cdot\left(\sum_{j=0}^{7} n_{h_{j}} \eta_{j}\right)\right) \cdot \lambda\right]-\mathcal{N}(\lambda) \mathcal{N}(\mu) \sum_{h=0}^{7} \Theta_{h_{i}} \eta_{h} \\
& =\sum_{h=0}^{7} \sum_{j=1}^{7}(\Theta_{h_{i}} \omega_{h_{j}}-e_{j} \underbrace{\Theta_{h_{i}} \omega_{h_{0}}}_{=0}) C_{j} .
\end{aligned}
$$

The underbraced expression $\Theta_{h_{i}} \omega_{h_{0}}$ vanishes, because we always have $\delta_{i 0}=0$, since $i \neq 0$.

In view of (8) the latter equation simplifies to

$$
\sum_{h=0}^{7}\left[\Theta_{h_{i}}\left(\mu \cdot\left(\sum_{j=0}^{7} n_{h_{j}} \eta_{j}\right)\right) \cdot \lambda\right]-\mathcal{N}(\lambda) \mathcal{N}(\mu) \sum_{h=0}^{7} \Theta_{h_{i}} \eta_{h}=C_{i} \operatorname{det}(W)
$$

Thus, for $i=1, \ldots, 7$ we obtain

$$
C_{i}=\frac{\sum_{h=0}^{7}\left[\Theta_{h_{i}}\left(\mu \cdot\left(\sum_{j=0}^{7} n_{h_{j}} \eta_{j}\right)\right) \cdot \lambda\right]-\mathcal{N}(\lambda) \mathcal{N}(\mu) \sum_{h=0}^{7} \Theta_{h_{i}} \eta_{h}}{\operatorname{det}(W)} .
$$

Now we are in position to calculate the traces of the octonionic Weierstraß' functions.

First of all we recall that

$$
\begin{aligned}
& \zeta_{\lambda, \mu}(z)-\frac{1}{(\mathcal{N}(\lambda) \mathcal{N}(\mu))^{3}} \zeta(z) \\
& =\frac{1}{(\mathcal{N}(\lambda) \mathcal{N}(\mu))^{3}} \sum_{v \in \mathcal{V}_{\lambda, \mu ; \Omega_{8}} \backslash\{0\}} \zeta(z+v)+\sum_{j=1}^{7} V_{\tau(j)}(z) C_{j}+C .
\end{aligned}
$$


Since $\zeta$ is an odd function, $\zeta_{\lambda, \mu}$ is an odd function, too. Consequently,

$$
\zeta_{\lambda, \mu}(z)-\frac{1}{(\mathcal{N}(\lambda) \mathcal{N}(\mu))^{3}} \zeta(z)=\mathcal{O}(z)
$$

around zero, because the singular parts cancel out, following from Proposition 4.11.

In view of $\lim _{z \rightarrow 0} \wp_{\tau(i)}(z)-q_{\tau(i)}(z)=0$ for all $i=1, \ldots, 7$, which is clear from the series representation, because $\lim _{z \rightarrow 0} q_{\tau(i)}(z+\omega)-q_{\tau(i)}(\omega)=0$, one even has that the expression on the right-hand side is of order $\mathcal{O}\left(z^{3}\right)$.

So, in particular,

$$
\lim _{z \rightarrow 0}\left(\zeta_{\lambda, \mu}(z)-\frac{1}{(\mathcal{N}(\lambda) \mathcal{N}(\mu))^{3}} \zeta(z)\right)=0,
$$

and since $\lim _{z \rightarrow 0} V_{\tau(j)}(z)=0$, one has

$$
\frac{1}{(\mathcal{N}(\lambda) \mathcal{N}(\mu))^{3}} \sum_{v \in \mathcal{V}_{\lambda, \mu ; \Omega_{8}} \backslash\{0\}} \zeta(v)=-C .
$$

Next,

$$
\frac{\partial \zeta_{\lambda, \mu}}{\partial x_{i}}-\frac{1}{(\mathcal{N}(\lambda) \mathcal{N}(\mu))^{3}} \wp_{\tau(i)}
$$

is an even expression and of the form $\mathcal{O}\left(z^{2}\right)$ around the origin. So,

$$
\lim _{z \rightarrow 0}\left(\frac{\partial \zeta_{\lambda, \mu}(z)}{\partial x_{i}}-\frac{1}{(\mathcal{N}(\lambda) \mathcal{N}(\mu))^{3}} \wp_{\tau(i)}(z)\right)=0 .
$$

On the other hand,

$$
\begin{aligned}
& \frac{\partial \zeta_{\lambda, \mu}(z)}{\partial x_{i}}-\frac{1}{(\mathcal{N}(\lambda) \mathcal{N}(\mu))^{3}} \wp_{\tau(i)}(z) \\
& =\frac{1}{(\mathcal{N}(\lambda) \mathcal{N}(\mu))^{3}} \sum_{v \in \mathcal{V}_{\lambda, \mu ; \Omega_{8}} \backslash\{0\}} \wp_{\tau(i)}(z+v)+\sum_{j=1}^{7} \frac{\partial}{\partial x_{i}} V_{\tau(j)}(z) C_{j} .
\end{aligned}
$$

Thus, with the same limit argument we obtain that

$$
\frac{1}{(\mathcal{N}(\lambda) \mathcal{N}(\mu))^{3}} \sum_{v \in \mathcal{V}_{\lambda, \mu ; \Omega_{8}} \backslash\{0\}} \wp_{\tau(i)}(v)=-C_{i} .
$$

If we apply the formula for the expression of the constants $C_{i}$ derived above, then we finally arrive at the desired trace formula for the octonionic CM-division values of the associated $\wp_{\tau(i)}$-function,

$$
\begin{aligned}
& \sum_{v \in \mathcal{V}_{\lambda, \mu ; \Omega_{8}} \backslash\{0\}} \wp_{\tau(i)}(v) \\
= & -\frac{(\mathcal{N}(\lambda) \mathcal{N}(\mu))^{3}}{\operatorname{det}(W)}\left[\sum_{h=0}^{7} \Theta_{h_{i}}\left(\mu \cdot\left(\sum_{j=0}^{7} n_{h_{j}} \eta_{j}\right) \cdot \lambda\right)-\mathcal{N}(\lambda) \mathcal{N}(\mu) \sum_{h=0}^{7} \Theta_{h_{i}} \eta_{h}\right]
\end{aligned}
$$

for each $i=1, \ldots, 7$, and the theorem is proved. 
Remark 4.17. For the sake of completeness we want to re-emphasize that the constant $C$ gives up to a minus sign multiplied with the third power of the quadratic norm expressions exactly the value of the trace of the CM-division values of the (O)-regular Weierstraß $\zeta$-function:

$$
\sum_{v \in \mathcal{V}_{\lambda, \mu ; \Omega_{8}} \backslash\{0\}} \zeta(v)=-(\mathcal{N}(\lambda) \mathcal{N}(\mu))^{3} \cdot C .
$$

Unfortunately, unlike for the constants $C_{i}$, we so far have no further information on the algebraic nature of the constant $C$.

\subsection{Final remark and outlook}

The trace of the octonionic division values of the functions $\wp_{\tau(i)}$ is an octonion whose real components are elements from the field that is generated by the algebraic number field of the real components of the primitive periods $\omega_{h}$ (canonically from a triquadratic number field) and by the real components of the octonionic Legendre constants $\eta_{h}$. It would be a great goal to figure out under which conditions these are algebraic and if one can do some class field theory with these octonionic functions.

Furthermore, it would be an essential question whether similar constructions could be carried over to the more general framework of Cayley-Dickson algebras or even more generally to graded deformed $\mathbb{R}_{F} \mathbb{Z}^{n}$-algebras that we discussed in [1]. As explained in Section 3, the theory of CM-lattices is available for this much more general setting. However, in the proofs and in the constructions of Section 4.3 we applied at several places the alternative property, which is true in the octonionic case but not anymore beyond this when proceeding with the usual Cayley-Dickson doubling. Perhaps some of these results can be carried over to the context of the algebra of the $2^{n}$-ons considered by D. Warren in [29], indicating another possibility for further future research in this kind of direction.

A possible field of application consists also in understanding whether these generalizations of elliptic functions may play a similar role in $G_{2}$-gauge theories, analogous to the role of quaternionic regular elliptic functions playing for $S U(2)$ instantons, cf. [13].

Another open problem, suggested by one of the referee's, would be the following. We have constructed a family of octonionic Weierstraß $\wp$ functions $\wp_{\tau(i)}$ $(i=1, \ldots, 7)$ from partial derivation of one octonionic Weierstraß $\zeta$-function. Actually, in complex analysis the Weierstraß $\zeta$-function, in turn, can be derived from one of the four Jacobi theta functions, namely, by $\wp(u)=-\frac{d^{2}}{d u^{2}} \log \theta(u)$. So, the Weierstraß $\zeta$-function can be constructed from that theta function as derivative and the Weierstraß $\wp$-function as its second derivative. It is an open question (even in the quaternionic case) whether one can define a monogenic theta function for a general lattice and if one can derive all the generalized Weierstraß $\wp$-functions from partial derivation of such a monogenic theta function (or perhaps of several ones). Note that partial derivation of a Weierstraß $\zeta$-function in the octonionic case gives seven linearly independent different Weierstraß $\wp_{\tau(i)}$ functions. Furthermore, if one introduces one primitive of the Weierstraß $\zeta$-function, then this function will also 
induce seven linearly independent Weierstraß $\zeta$-functions. One among them might be the particular one described in this paper. Each of these Weierstraß $\zeta$-functions, in turn, induces seven Weierstraß $\wp$-functions by partial derivation.

\section{Acknowledgement}

The author is very thankful to the referees of this paper. Their suggestions and comments were extremely valuable and contributed a lot to the successful development of this paper.

Funding. Open Access funding enabled and organized by Projekt DEAL.

Open Access. This article is licensed under a Creative Commons Attribution 4.0 International License, which permits use, sharing, adaptation, distribution and reproduction in any medium or format, as long as you give appropriate credit to the original author(s) and the source, provide a link to the Creative Commons licence, and indicate if changes were made. The images or other third party material in this article are included in the article's Creative Commons licence, unless indicated otherwise in a credit line to the material. If material is not included in the article's Creative Commons licence and your intended use is not permitted by statutory regulation or exceeds the permitted use, you will need to obtain permission directly from the copyright holder. To view a copy of this licence, visit http://creativecommons.org/licenses/by/4.0/.

Publisher's Note. Springer Nature remains neutral with regard to jurisdictional claims in published maps and institutional affiliations.

\section{References}

[1] H. Albuquerque, R.S. Kraußhar. Multiplicative invariant lattices in $\mathbb{R}^{n}$ obtained by twisting of group algebra and some explicit characterizations. Journal of Algebra $\mathbf{3 1 9}$ No. 3 (2008), 1116-1131.

[2] J. Baez. The octonions. Bull. Amer. Math. Soc. 39 (2002), 145-205.

[3] F. Brackx, R. Delanghe, F. Sommen. Clifford Analysis, Pitman Res. Notes in Math., $76,1982$.

[4] F. Colombo, I. Sabadini, D. C. Struppa. Entire slice regular functions. Springer Briefs in Mathematics. Springer, Cham, 2016. v+118 pp.

[5] R. Delanghe, F. Sommen, V. Souček. Clifford Algebra and Spinor-valued Functions. Mathematics and Its Applications 53, Kluwer Academic Publishers, 1992.

[6] P. Dentoni, M. Sce. Funzioni regolari nell'algebra di Cayley. Rend. Sem. Mat. Univ. Padova 50 (1973), 251--267.

[7] C. Flaut, V. Shpakivski. Holomorphic functions in generalized Cayley-Dickson algebras. Adv. Appl. Clifford Algebras 25 (2015), 95--112.

[8] E. Freitag. Complex Analysis 2. Springer, Heidelberg, 2011.

[9] E. Frenod, S. V. Ludkowski. Integral operator approach over octonions to solution of nonlinear PDE. Far East Journal of Mathematical Sciences (FJMS) (2017). 
[10] R. Fueter. Functions of a Hypercomplex Variable. Lecture Notes written and supplemented by E. Bareiss, ETH Zürich, Wintersemester 1948/49.

[11] R. Ghiloni, A. Perotti. Slice regular functions on real alternative algebras. Adv. Math. 226 (2011), 1662-1691.

[12] K. Gürlebeck, K. Habetha, W. Sprößig. Holomorphic Functions in the Plane and ndimensional space. Birkhäuser, Basel, 2008.

[13] F. Gürsey, H. Tze. On the role of division and Jordan algebras in particle physics. World Scientific, Singapore, 1996.

[14] D. Hilbert. The Theory of Algebraic Number Fields (Zahlbericht 1896). Springer, Berlin, 1998.

[15] L. Hörmander. An Introduction to Complex Analysis in Several Variables. NorthHolland Mathematical Library, Vol. 7. North-Holland, Amsterdam, 1990.

[16] K. Imaeda. Sedenions: algebra and analysis. Appl. Math. Comp. 115 (2000), 77-88.

[17] M. Jin, G. Ren, I. Sabadini. Slice Dirac operator over octonions. Isr. J. Math. (2020). https://doi.org/10.1007/s11856-020-2067-z

[18] J. Kauhanen, H. Orelma. Cauchy-Riemann Operators in Octonionic Analysis. Advances in Applied Clifford Algebras 28 No. 1 (2018), 14pp.

[19] J. Kauhanen, H. Orelma. On the structure of Octonion regular functions. Advances in Applied Clifford Algebra 29 No. 4 (2019), 17pp.

[20] R.S. Kraußhar. The multiplication of the Clifford-analytic Eisenstein series. Journal of Number Theory 102 No. 2 (2003), 353-382.

[21] R.S. Kraußhar. Generalized automorphic Forms in hypercomplex spaces. Birkhäuser, Basel, 2004.

[22] S. Lang. Complex multiplication. Grundlehren der Mathematischen Wissenschaften (Fundamental Principles of Mathematical Sciences) 255 Springer, New York, 1983.

[23] H.R. Malonek. Rudolf Fueter and his motivation for hypercomplex function theory. Advances in Applied Clifford Algebras 11 (2001), 219-229.

[24] W. Nef. Die unwesentlichen Singulariäten der regulären Funktionen einer Quaternionenvariablen. Comm. Math. Helv. 16 (1943-44), 284-304.

[25] C. Nolder. Much to do about octonions. AIP Proceedings, ICNAAM 2018, 2116, 160007 (2019); https://doi.org/10.1063/1.5114151

[26] K. Nono. On the octonionic linearization of Laplacian and octonionic function theory. Bull. Fukuoka Univ. Ed. Part III 37 (1988), 1-15.

[27] J. Ryan. Clifford Analysis with generalized elliptic and quasi-elliptic functions. Applicable Analysis, 13 (1982), 151-171.

[28] B. Schoeneberg. Elliptic Modular Functions. Die Grundlagen der mathematischen Wissenschaften 201, Springer, Berlin-Heidelberg-New York, 1974.

[29] W. D. Smith. Quaternions, octonions, 16-ons and $2^{n}$-ons; New kinds of numbers. Pensylvania State University (2004), 1-68. DOI: 10.1.1.672.2288

[30] Xing-min Li and Li-Zhong Peng. On Stein-Weiss conjugate harmonic function and octonion analytic function. Approx. Theory and its Appl. 16 (2000), 28-36.

[31] Xing-min Li, K. Zhao, Li-Zhong Peng. The Laurent series on the octonions. Advances in Applied Clifford Algebras 11 (S2) (2001), 205-217. 
[32] Xing-min Li and Li-Zhong Peng. The Cauchy integral formulas on the octonions. Bull. Belg. Math. Soc. 9 (2002), 47-62.

[33] Xing-min Li , Zhao Kai, Li-Zhong Peng. Characterization of octonionic analytic functions. Complex Variables 50 No. 13 (2005), 1031-1040.

Rolf Sören Kraußhar

Fachbereich Fachwissenschaft Mathematik

Erziehungswissenschaftliche Fakultät

Universität Erfurt

Nordhäuser Str. 63

D 99089 Erfurt

Germany

e-mail: soeren.krausshar@uni-erfurt.de

Submitted: December 10, 2019.

Revised: October 14, 2020.

Accepted: October 28, 2020. 